ARTICLE

DOI: $10.1038 / s 41467-018-02953-2 \quad$ OPEN

\title{
Laboratory evidence of dynamo amplification of magnetic fields in a turbulent plasma
}

\author{
P. Tzeferacos ${ }^{1,2}$, A. Rigby (10 1 , A. F. A. Bott ${ }^{1}$, A.R. Bell1', R. Bingham³,4, A. Casner 5 , F. Cattaneo², E.M. Churazov ${ }^{6,7}$, \\ J. Emig ${ }^{8}$, F. Fiuza ${ }^{9}$, C.B. Forest ${ }^{10}$, J. Foster ${ }^{11}$, C. Graziani ${ }^{2}$, J. Katz ${ }^{12}$, M. Koenig ${ }^{13}$, C.-K. Li ${ }^{14}$, J. Meinecke , \\ R. Petrasso ${ }^{14}$, H.-S. Park ${ }^{8}$, B.A. Remington ${ }^{8}$, J.S. Ross ${ }^{8}$, D. Ryu (D) ${ }^{15}$, D. Ryutov ${ }^{8}$, T.G. White ${ }^{1}$, B. Reville (D) ${ }^{16}$, \\ F. Miniati ${ }^{17}$, A.A. Schekochihin', D.Q. Lamb², D.H. Froula ${ }^{12} \&$ G. Gregori (i) ${ }^{1,2}$
}

Magnetic fields are ubiquitous in the Universe. The energy density of these fields is typically comparable to the energy density of the fluid motions of the plasma in which they are embedded, making magnetic fields essential players in the dynamics of the luminous matter. The standard theoretical model for the origin of these strong magnetic fields is through the amplification of tiny seed fields via turbulent dynamo to the level consistent with current observations. However, experimental demonstration of the turbulent dynamo mechanism has remained elusive, since it requires plasma conditions that are extremely hard to re-create in terrestrial laboratories. Here we demonstrate, using laser-produced colliding plasma flows, that turbulence is indeed capable of rapidly amplifying seed fields to near equipartition with the turbulent fluid motions. These results support the notion that turbulent dynamo is a viable mechanism responsible for the observed present-day magnetization.

\footnotetext{
${ }^{1}$ Department of Physics, University of Oxford, Parks Road, Oxford OX1 3PU, UK. ${ }^{2}$ Department of Astronomy and Astrophysics, University of Chicago, 5640 S. Ellis Ave, Chicago, IL 60637, USA. ${ }^{3}$ Rutherford Appleton Laboratory, Chilton, Didcot OX11 OQX, UK. ${ }^{4}$ Department of Physics, University of Strathclyde, Glasgow G4 ONG, UK. ${ }^{5}$ CEA, DAM, DIF, 91297 Arpajon, France. ${ }^{6}$ Max Planck Institute for Astrophysics, Karl-Schwarzschild-Strasse 1, 85741 Garching, Germany. ${ }^{7}$ Space Research Institute (IKI), Profsouznaya 84/32, Moscow 117997, Russia. ${ }^{8}$ Lawrence Livermore National Laboratory, Livermore, CA 94550, USA. ${ }^{9}$ SLAC National Accelerator Laboratory, 2575 Sand Hill Road, Menlo Park, CA 94025, USA. ${ }^{10}$ Physics Department, University of Wisconsin-Madison, 1150 University Avenue, Madison, WI 53706, USA. ${ }^{11}$ AWE, Aldermaston, Reading, West Berkshire RG7 4PR, UK. ${ }^{12}$ Laboratory for Laser Energetics, University of Rochester, 250 E. River Rd, Rochester, NY 14623, USA. ${ }^{13}$ Laboratoire pour I'Utilisation de Lasers Intenses, UMR7605, CNRS CEA, Université Paris VI Ecole Polytechnique, 91128 Palaiseau Cedex, France. ${ }^{14}$ Massachusetts Institute of Technology, Cambridge, MA 02139, USA. ${ }^{15}$ Department of Physics, UNIST, Ulsan 689-798, Korea. ${ }^{16}$ School of Mathematics and Physics, Queens University Belfast, Belfast BT7 1NN, UK. ${ }^{17}$ Department of Physics, ETH Zürich, Wolfgang-Pauli-Strasse 27, Zürich CH-8093, Switzerland. Correspondence and requests for materials should be addressed to G.G. (email: gianluca.gregori@physics.ox.ac.uk)
} 
$\mathrm{D}$ iffuse radio-synchrotron emission observations and Faraday rotation measurements ${ }^{1}$ have revealed magnetic field strengths ranging from a few $n G$ and tens of $\mu \mathrm{G}$ in extragalactic disks, halos and clusters, up to hundreds of TG in magnetars, as inferred from their spin-down ${ }^{2}$. That turbulence is of central importance in the generation and evolution of magnetic fields in the Universe is widely accepted ${ }^{3}$. Plasma turbulence can be found in myriads of astrophysical objects, where it is excited by a range of processes: cluster mergers, supernovae explosions, stellar outflows, etc. ${ }^{4-7}$. If a turbulent plasma is threaded by a weak magnetic field, the stochastic motions of the fluid will stretch and fold this field, amplifying it until it becomes dynamically significant ${ }^{8,9}$. According to the current standard picture, the amplification can be summarized in two basic steps ${ }^{10,11}$. First, when the initial field is small the magnetic energy grows exponentially (kinematic phase). This phase terminates when the magnetic energy reaches approximate equipartition with the kinetic energy at the dissipation scale. Beyond this point, the magnetic energy continues to grow linearly in time (nonlinear phase) until, after roughly one outer-scale eddy-turnover time, it saturates at a fraction of the total kinetic energy of the fluid motions ${ }^{10,12}$. This is what is referred to as the turbulent dynamo mechanism for magnetic field amplification.
The seed fields that the dynamo amplifies can be produced by a variety of different physical processes. In many astrophysical environments where the plasma is initially unmagnetized, and most certainly at the time when proto-galaxies were forming, baroclinic generation of magnetic fields due to misaligned density and temperature gradients - the Biermann battery mechanismcan provide initial seeds ${ }^{13}$. The same starting fields also occur in laser-produced plasmas ${ }^{14,15}$

Theoretical expectations that turbulent dynamo must operate go back more than half a century, ${ }^{8,9,16}$ and the first direct numerical confirmation of this effect was achieved 35 years ago ${ }^{17}$. A significant body of theoretical work has developed over the years ${ }^{18-20}$ that has greatly expanded our understanding of the mechanism-for recent reviews of the current state of affairs see refs. 21 and 22 . Despite these advancements, demonstrating turbulent dynamo amplification in the laboratory has remained elusive. This is primarily because of the difficulty of achieving experimentally magnetic Reynolds numbers $\left(\mathrm{Rm}=u_{L} L / \mu\right.$, where $u_{L}$ is the flow velocity at the outer scale $L$, and $\mu$ is the magnetic diffusivity) above the critical threshold of a few hundred required for dynamo ${ }^{23}$. Such a demonstration would not only establish experimentally the soundness of the existing theoretical and numerical expectations for one of the most fundamental physical

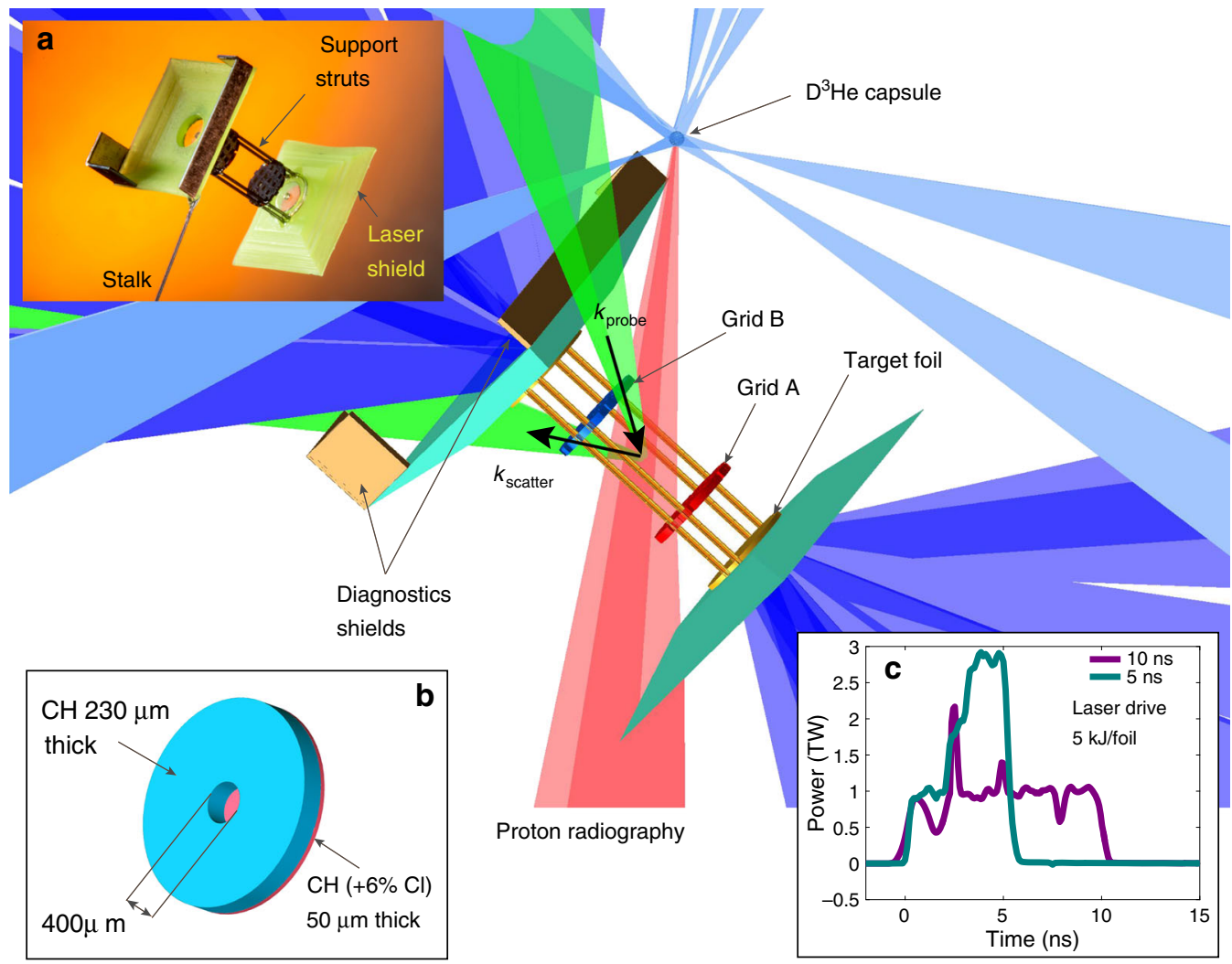

Fig. 1 Experimental configuration. The main target (see photo in a) consists of two $\mathrm{CH}$ foils doped with $6 \%$ chlorine in atomic number (b) that are separated by $8 \mathrm{~mm}$. Each foil is illuminated by ten $500 \mathrm{~J}, 1 \mathrm{~ns}$ pulse length, frequency tripled ( $351 \mathrm{~nm}$ wavelength) laser beams with $800 \mu \mathrm{m}$ spot diameter. The beams are stacked in time to achieve the two pulse profiles shown in c. An additional set of 17 beams, all fired simultaneously, are used to implode a $420 \mu \mathrm{m}$ diameter capsule consisting of a 2 - $\mu \mathrm{m}$-thick $\mathrm{SiO}_{2}$ shell filled with $\mathrm{D}_{2}$ gas at $6 \mathrm{~atm}$ and ${ }^{3} \mathrm{He}$ at 12 atm. The implosion produces mono-energetic protons at 3.3 and $15 \mathrm{MeV}$ with $\sim 40 \mu \mathrm{m}$ diameter source size, which traverse the plasma and are then collected by a CR-39 nuclear track detector with a total magnification factor of 28. The plasma expansion towards the center of the target is perturbed by the presence of two grids, placed 4 mm apart, with a $300 \mu \mathrm{m}$ hole width and $300 \mu \mathrm{m}$ hole spacing. Grid A has the central hole aligned on the center axis connecting the two foils, while grid B has the hole pattern shifted so that the central axis crosses the middle point between two holes. Thomson scattering uses a $30 \mathrm{~J}, 1 \mathrm{~ns}$, frequency doubled (wavelength $\lambda$ $=526.5 \mathrm{~nm}$ ) laser beam to probe the plasma on the axis of the flow, $400 \mu \mathrm{m}$ from the center and in a $50 \mu \mathrm{m}$ focal spot, towards grid B. The scattered light is collected with $63^{\circ}$ scattering angle and the geometry is such that the scattering wavenumber $k=k_{\text {scatter }}-k_{\text {probe, }}$, where $\left|k_{\text {scatter }}\right| \approx\left|k_{\text {probe }}\right|=2 \pi / \lambda$, is parallel to the axis of the flow 
a

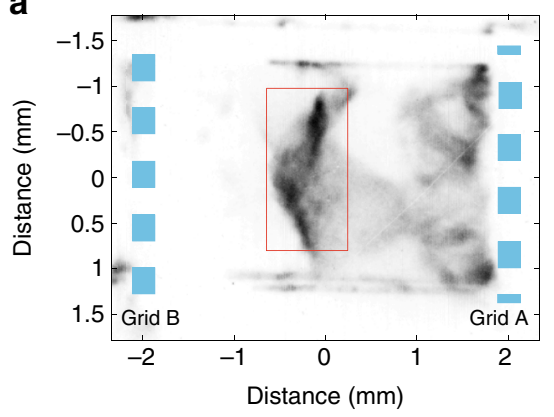

C

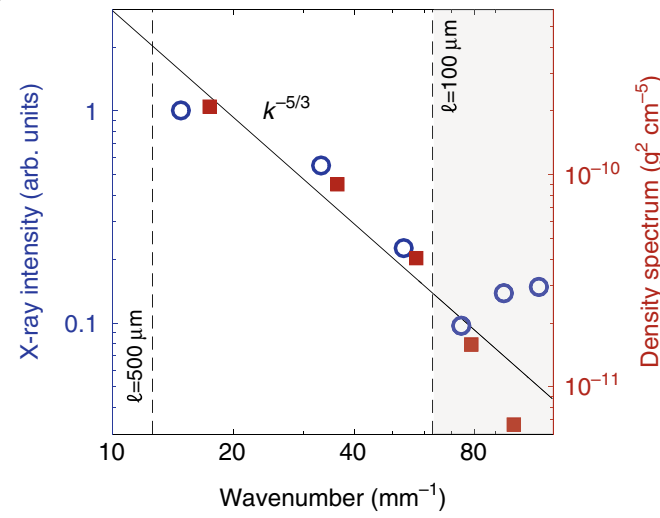

b

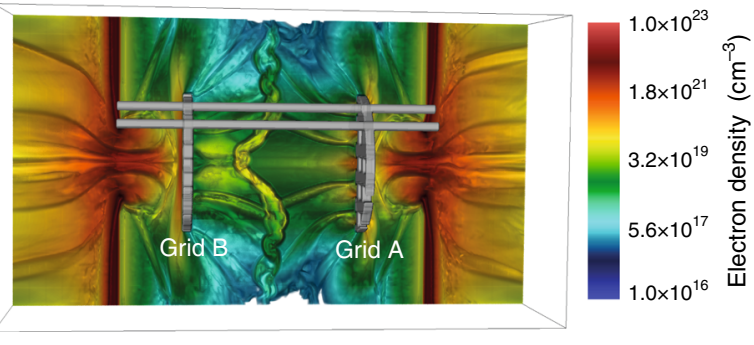

d

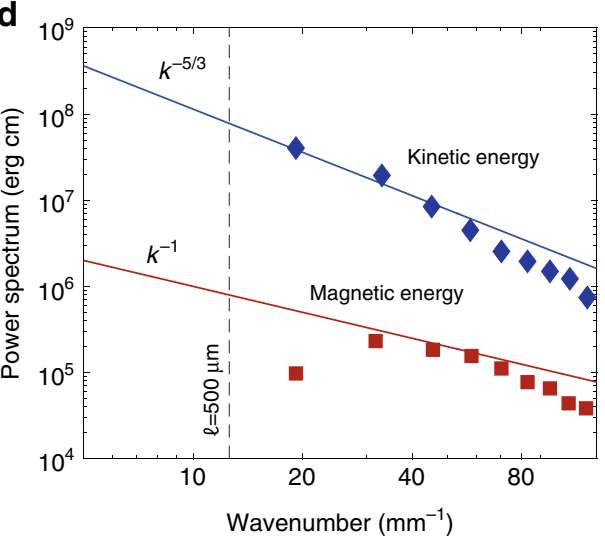

Fig. 2 Characterization of the plasma turbulence. a X-ray pinhole image of the colliding flows at $t=35 \mathrm{~ns}$ after the laser drive, using the $5 \mathrm{~ns}$ pulse profile. The image was recorded onto a framing camera with $\sim \mathrm{ns}$ gate width and filtered with $0.5 \mu \mathrm{m} \mathrm{C}_{2} \mathrm{H}_{4}$ and $0.15 \mu \mathrm{m}$ Al. The pinhole diameter is $50 \mu \mathrm{m}$. $\mathbf{b}$ Rendering of the electron density from three-dimensional FLASH simulations at $t=35 \mathrm{~ns}$. c The open blue circles give the power spectrum of the X-ray emission from the collision region, defined by the rectangular region shown in panel $\mathbf{a}$. The power spectrum has been filtered to remove edge effects and image defects. Details of this procedure are given in Supplementary Methods. The shaded region at high wavenumbers is dominated by noise. The spectrum of the density fluctuations, as obtained from FLASH simulations in the turbulent region, is shown with red squares. $\mathbf{d}$ Blue diamonds: power spectrum of the kinetic energy from FLASH simulations. Red squares: power spectrum of magnetic energy from FLASH simulations. The simulated magnetic energy spectrum is considerably shallower than the Kolmogorov-like kinetic energy spectrum, as predicted by ref. ${ }^{23}$ and other studies in the Pm $<1$ regime (see text)

processes in astrophysics ${ }^{24}$, but also provide a platform ${ }^{25}$ to investigate other fundamental processes that require a turbulent magnetized plasma, such as particle acceleration and reconnection.

To date, experimental investigation of magnetic field amplification has primarily been carried out in liquid-metal experiments, such as the von Kármán swirling flow of ref. ${ }^{26}$ and the Riga dynamo experiment ${ }^{27,28}$ inspired by the Ponomarenko dynamo $^{29}$. The driven dynamos achieved in these experiments depended on a particular fluid flow rather than a purely turbulent effect leading to a stochastic field. More recent work has focused on laser-driven plasmas ${ }^{25,30,31}$, but studying a regime that is a precursor to dynamo, because of the modest magnetic Reynolds numbers that could be achieved.

In the following, we describe experiments in which we reach magnetic Reynolds numbers above the expected dynamo threshold. We detail the experimental configuration and present diagnostic measurements that fully characterize the plasma state of the magnetized turbulence. By utilizing two independent magnetic field diagnostics we are able to demonstrate the turbulent dynamo amplification of seed magnetic fields to dynamical equipartition with the kinetic energy of the turbulent motions.

\section{Results}

Experimental platform. The experiments were performed at the Omega laser facility at the Laboratory for Laser Energetics of the University of Rochester ${ }^{32}$ using a combined platform that builds on our previous work on smaller laser facilities ${ }^{15,30,31}$. Laser ablation of a chlorine-doped plastic foil launches a plasma flow from its rear surface. The plasma then passes through a solid grid and collides with an opposite moving flow, produced in the same manner. In order to increase the destabilization of the motions as the flows collide, the two grids have hole patterns that are shifted with respect to each other. Further details on the experimental setup are given in Fig. 1. A set of diagnostics has been fielded to measure the properties of the flow, its turbulence and the magnetic field generated by it (see Figs. 2 and 3).

Extensive two-dimensional and three-dimensional simulations done prior to the experiments using the radiationmagnetohydrodynamics (MHD) code FLASH informed their design (see Fig. 2b and Supplementary Methods), including the details of the targets and the grids, and the timing of the diagnostics 33,34 .

Characterization of the turbulent plasma. X-ray emission can be used to characterize the interaction of the colliding flows and assess properties of the resulting plasma inhomogeneities. The presence of a small amount of chlorine in the plasma enhances the emission in the soft wavelength region $(<2 \mathrm{keV})$. Soft X-ray images taken at $t=35 \mathrm{~ns}$ from the start of the laser drive, which is after the flows collide, indicate a broad non-uniform spatial distribution of the emission over a region more than $1 \mathrm{~mm}$ across.

In order to characterize the state of the turbulent plasma produced by the collision of two laser-produced jets (as shown in 
Fig. 1), power spectra of the X-ray intensity fluctuations were extracted from the experimental data using a two-dimensional fast Fourier transform (see Fig. 2a, c). Under the assumption of isotropic statistics, fluctuations in the detected X-ray intensity are directly related to density fluctuations (see discussion in Supplementary Methods). The power spectrum of the density fluctuations extracted from the X-ray data is consistent with a Kolmogorov power law ( $k^{-5 / 3}$ scaling). Experimental data from other diagnostics indicate that the plasma motions are mainly subsonic (Mach number $\lesssim 1$ at the outer scale); as a result, density fluctuations injected at large scales behave as a passive scalar and the spectra of the density and velocity fluctuations should be the same $e^{35,36}$. We conclude that the X-ray emission supports the notion that turbulent motions are present in the interaction region. This is also confirmed by FLASH simulations ${ }^{34}$, which predict subsonic motions of the plasma following the flow collision. Furthermore, the power spectrum of density and velocity fluctuations can be calculated directly from FLASH, and the results are consistent the same power law scaling for both (Fig. 2c, d).

The Thomson scattering diagnostic (see Fig. 1 and Supplementary Methods) allows us to measure simultaneously three different velocities associated with the flow ${ }^{37}$. First, the bulk plasma flow velocity-composed of a mean flow velocity $U$ and
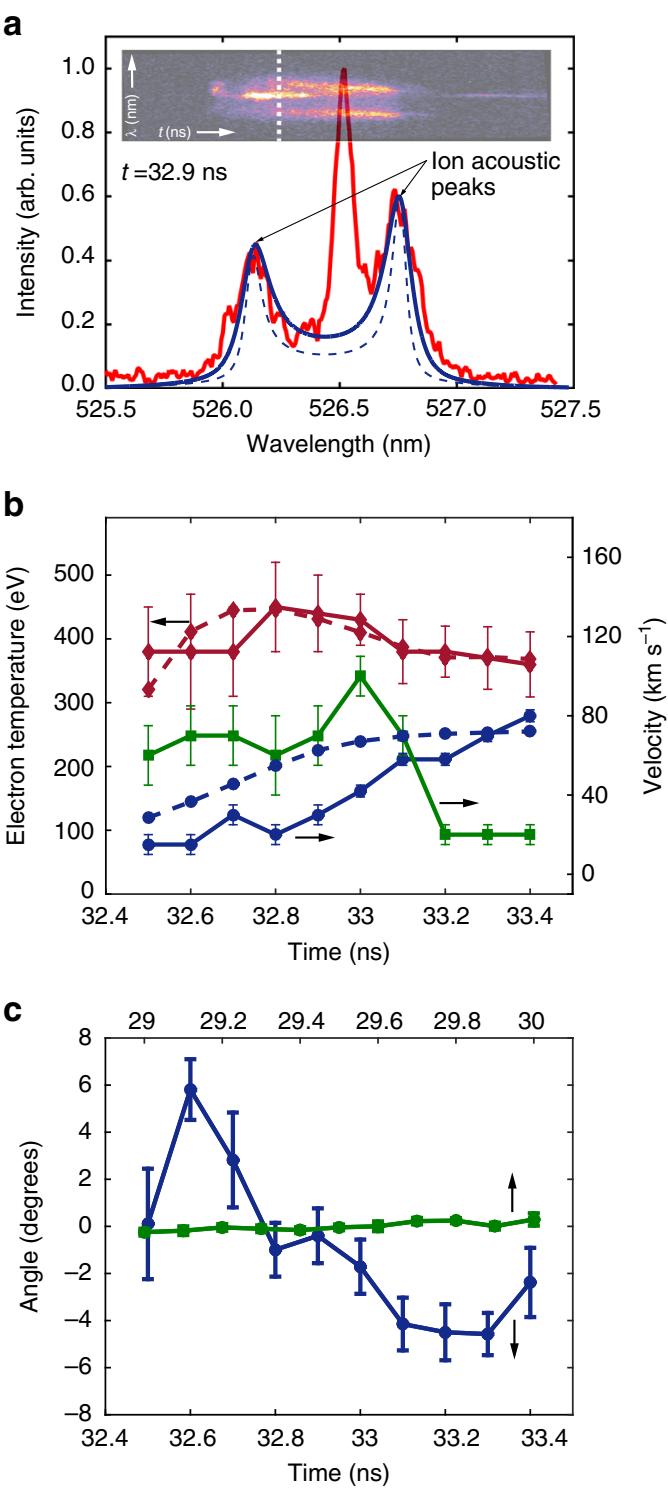

outer-scale turbulent velocity $u_{L}$-is obtained from the measurements of blueshifts (in frequency) of the scattered light resulting from the bulk plasma moving towards grid B. Second, the separation of the ion-acoustic waves is an accurate measure of the sound speed and thus of the electron temperature, $T_{e}$. Third, the FLASH prediction of equal ion and electron temperatures allows us to infer from the broadening of the ion-acoustic features the turbulent velocity $u_{\ell}$ on the scale $\ell \sim 50 \mu \mathrm{m}$ (the Thomson scattering focal spot) ${ }^{31,38}$.

Based on these measurements, we find the following. Before the collision, the two plasma flows move towards each other with axial mean velocity $U \leqslant 200 \mathrm{~km} \mathrm{~s}^{-1}$ in the laboratory rest frame, and have an electron temperature $T_{\mathrm{e}} \approx 220 \mathrm{eV}$ (see Supplementary Figure 1). After the collision, the axial flow slows down to 20-40 $\mathrm{km} \mathrm{s}^{-1}$, with motions being converted into transverse components. The electron temperature increases considerably, reaching $T_{\mathrm{e}} \approx 450 \mathrm{eV}$ (Fig. 3). The measured time-averaged (RMS) turbulent velocity at scale $\ell$ is $u_{\ell} \sim 55 \mathrm{~km} \mathrm{~s}^{-1}$. If $u_{\ell}$ has Kolmogorov scaling, the turbulent velocity at the outer scale must therefore be $u_{L} \sim u_{\ell}(L / \ell)^{1 / 3} \approx 100 \mathrm{~km} \mathrm{~s}^{-1}$. Electron density estimates can be obtained from the measured total intensity of the Thomson scattered radiation, to give a value $n_{\mathrm{e}} \approx 10^{20} \mathrm{~cm}^{-3}$, which is also consistent with values predicted by FLASH simulations ${ }^{34}$. As shown in Supplementary Methods, plasmas with these parameters can be well described as being collisional, and in the resistive MHD regime.

For an MHD-type plasma, we can estimate the characteristic fluid and magnetic Reynolds numbers attained in our experiment. We find $\operatorname{Re}=u_{L} L / \nu \sim 1200$ ( $\nu$ is the viscosity), and $\mathrm{Rm} \sim 600$, using $L \sim 600 \mu \mathrm{m}$, the characteristic driving scale determined by the average separation between grid openings. We have thus achieved conditions where $\mathrm{Rm}$ is comfortably larger than the expected critical magnetic Reynolds number required for turbulent

Fig. 3 Thomson scattering measurements. Electron temperatures and flow velocities are obtained by fitting the experimental data with the frequencydependent Thomson scattering cross-section ${ }^{37}$. In the fitting procedure we assumed an electron density of $\lesssim 10^{20} \mathrm{~cm}^{-3}$ (as predicted by FLASH simulations). At these electron densities, the frequency distribution of the scattered light does not depend on the electron density, which only provides an overall normalization factor. a Thomson scattering data (red solid line) at $t=32.9 \mathrm{~ns}$ obtained from a target driven with the $5 \mathrm{~ns}$ pulse profile. The blue dashed line corresponds to plasma in thermodynamic equilibrium (assuming equal electron and ion temperatures). The central peak is due to stray light at the probe laser wavelength (and it is used to determine the instrumental resolution of the spectrometer). The blue solid line corresponds to the case in which additional broadening due to turbulence is included in the fitting procedure. The inset in the top panel shows the time-streaked image of the Thomson scattered light. The resolution of the streak camera is $\sim 50$ ps and the Thomson scattering signal is fitted every 100 ps. b Flow velocity towards grid B (full blue circles), turbulent velocity (full green squares), and electron temperature (full red diamonds) as measured by Thomson scattering for the case of a target driven with the $5 \mathrm{~ns}$ laser profile. FLASH simulation results for the electron temperature and flow velocity in the probe volume are also reported in dashed lines. The error bars are estimated from the $\chi^{2}$ fit of the data. $\mathbf{c}$ Estimated Faraday rotation data from the Thomson scattering data. This was done by separating the scattered light into two orthogonal polarizations (see Supplementary Methods). The blue line corresponds to the same conditions as $\mathbf{b}$, above. The green line was obtained from an experiment involving a single-flow, single-grid experiment only, when the magnetic field is expected to be significantly smaller (see Supplementary Figure 8 for the proton radiography results arising for a single-flow, singlegrid experiment). The errors are determined by the standard deviation of the data within the shot 
dynamo $^{23}$. The experiment also lies in the regime where the magnetic Prandtl number is $\mathrm{Pm} \equiv \mathrm{Rm} / \mathrm{Re}<1$.

Magnetic field measurements. Magnetic fields were inferred using both Faraday rotation (Fig. 3) and proton radiography (Fig. 4). The rotation of the polarization angle of Thomson scattered light provides a measure of the variation of the
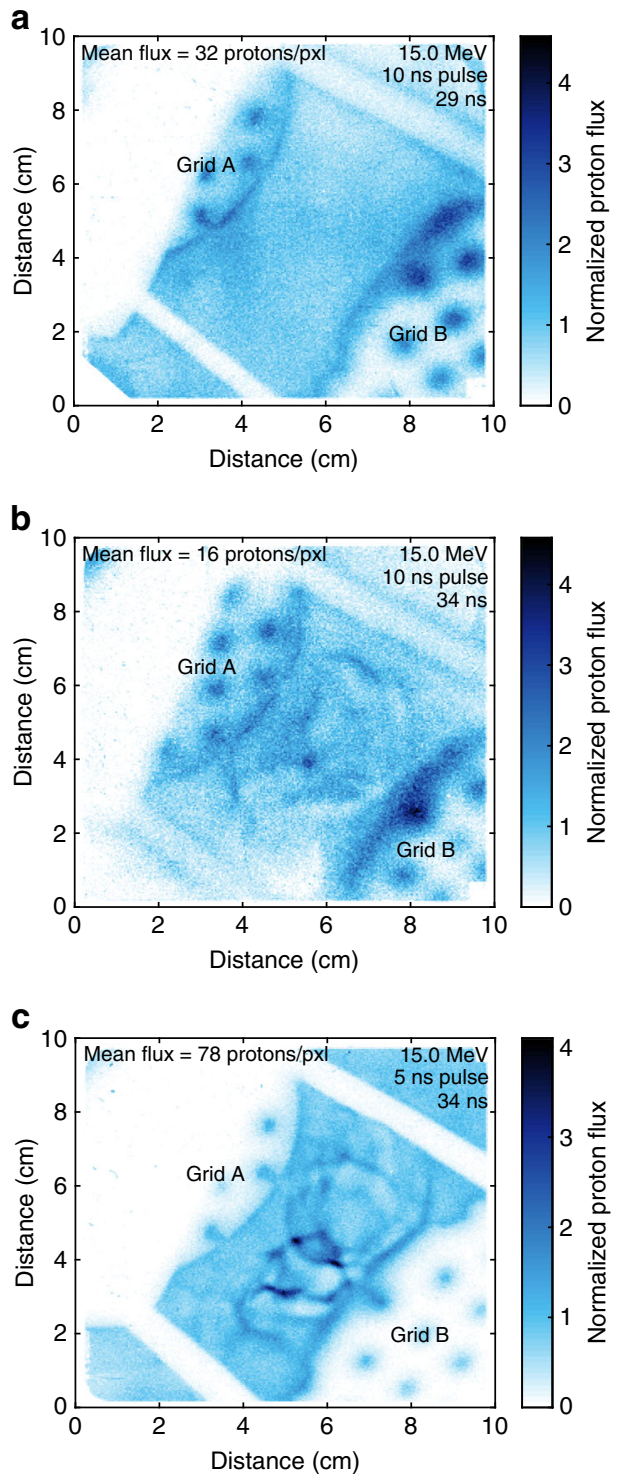

longitudinal component of the magnetic field integrated along the beam path, weighted by the electron density. Assuming a random field with correlation length $\ell_{B}$, we estimate $B_{\|, \mathrm{rms}} \approx 120\left(\Delta \theta / 3^{\circ}\right)$ $\left(n_{\mathrm{e}} / 10^{20} \mathrm{~cm}^{-3}\right)^{-1}\left(\ell_{n} \ell_{B} / 0.2 \mathrm{~mm}^{2}\right)^{-1 / 2} \mathrm{kG}$, where $B_{\|, \mathrm{rms}}$ is the root mean square (RMS) value of the magnetic field component parallel to the probe beam, $\Delta \theta$ is the rotation angle, and $\ell_{n} \sim L \approx$ $0.6 \mathrm{~mm}$ is the scale length of the electron density along the line of
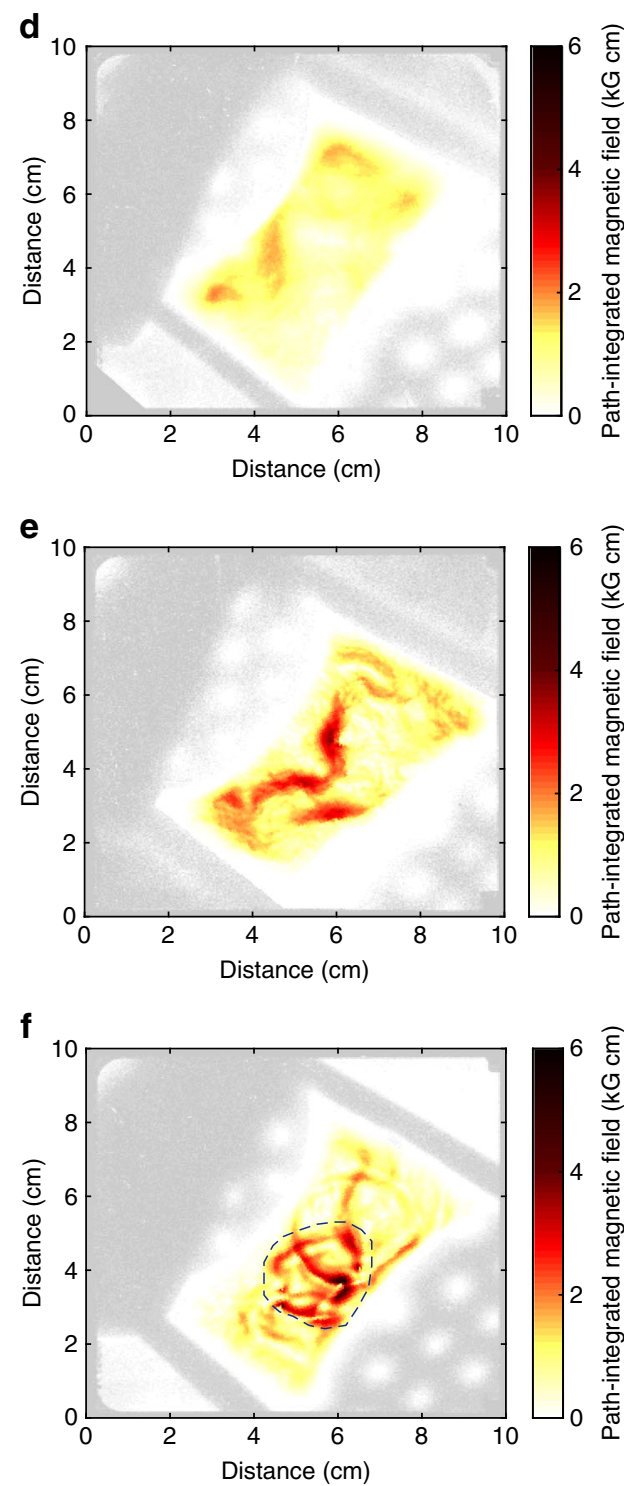

g

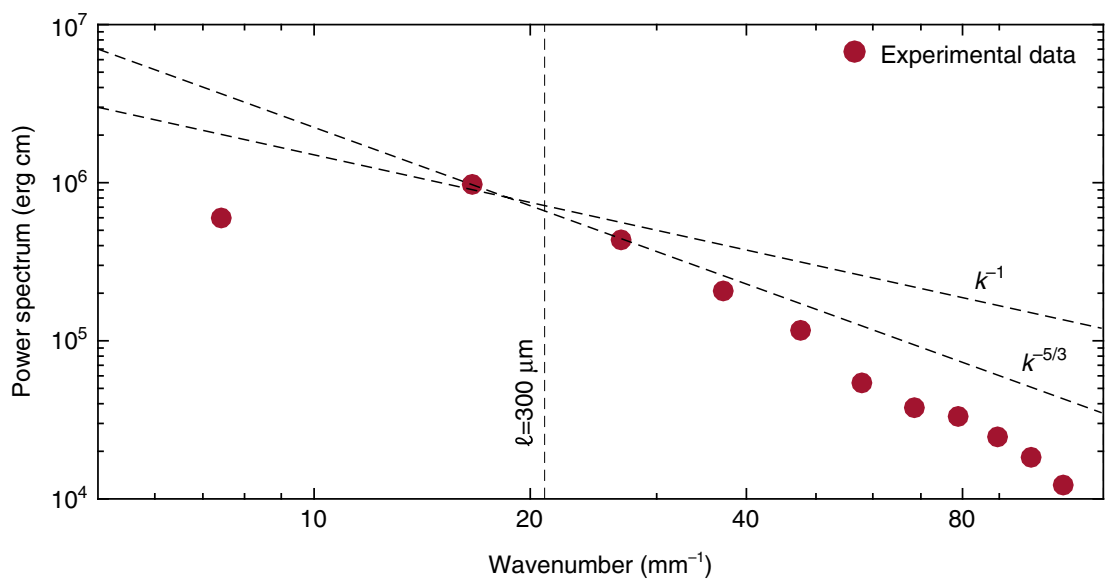


sight. Estimating $\ell_{B}$ is more challenging, but as a reasonable estimate we can take the size of the grid aperture $\left(\ell_{B} \sim 300 \mu \mathrm{m}\right)$. The choice of $\ell_{n} \ell_{B}$ was corroborated by the FLASH simulations via synthetic Faraday rotation measurements (see Supplementary Figure 11 and the discussion in Supplementary Methods).

The appearance of strong, sharp features in proton radiographs provides independent evidence for large magnetic fields ${ }^{39,40}$. Such structures are the result of initially divergent proton rays produced by an imploding $\mathrm{D}^{3} \mathrm{He}$ capsule being focused by magnetic forces as they traverse the plasma. This leads, at the detector plane, to localized regions where the proton counts greatly exceed their average value and other regions where they are strongly depleted. The detailed spatial structure of the pathintegrated magnetic field can be reconstructed from the experimental images assuming that the protons undergo small deflections as they pass through the plasma, and that the paths of neighboring protons do not cross before reaching the screen. Assuming isotropic statistics, this is sufficient information to calculate the power spectrum of the magnetic energy $E_{B}(k)$, and then the RMS magnetic field strength $B_{\text {rms }}$ of fluctuating fields via $B_{\mathrm{rms}}^{2}=8 \pi \int \mathrm{d} k E_{B}(k)$ (see refs. ${ }^{41,42}$ and Supplementary Methods). Figure 4 a shows that the magnetic field during the early phases of the collision is small, as no strong flux features appear in the radiographic image. The corresponding reconstructed RMS magnetic field strength $B_{\text {rms }}$ obtained from Fig. $4 \mathrm{~d}$ gives $B_{\mathrm{rms}} \lesssim 4 \mathrm{kG}$.

\section{Discussion}

Magnetic fields before the collision, and in the absence of any strong turbulence, are presumably Biermann battery fields produced at the laser spots and then advected by the flow, as indeed is confirmed by FLASH simulations. In contrast, Fig. $4 \mathrm{~b}, \mathrm{c}-$ corresponding to a later stage of the turbulent plasma's lifetime for the 10 and $5 \mathrm{~ns}$ pulse shapes, respectively-do indeed show strong features, indicative of increased fields strengths and altered morphology. These strong features are absent in single-flow experiments (see Supplementary Figure 8 in Supplementary Methods), suggesting that the interaction of the counterpropagating flows and subsequent development of turbulence is essential for magnetic field amplification. The reconstruction algorithm can also be applied to the images in Fig. $4 \mathrm{~b}$, c (see Fig. $4 \mathrm{e}, \mathrm{f}$ ); in the latter case, we obtain $B_{\text {rms }} \approx 100 \mathrm{kG}$ (see Supplementary Methods). This is consistent with our previous estimates based on Faraday rotation. We claim that this increase of the magnetic field during the collision cannot be simply explained by the compression of the field lines due to the formation of shocks (this would only account for a factor of two increase at most), nor by further generation by Biermann battery as the temperature gradients are not strong enough. This view is supported by FLASH simulations (see Supplementary Methods). Collisionless (magnetic field-generating) plasma processes such as the Weibel or filamentation instabilities cannot be responsible for the amplification of the magnetic field either, on account of the plasma's high collisionality (see Supplementary Methods).
In Fig. $4 \mathrm{~g}$ we show the spectrum of the magnetic energy, $E_{B}(k)$, calculated from the reconstructed path-integrated magnetic field. This is the spectrum on which the estimate of $B_{\text {rms }}$ is based. The peak of this spectrum occurs at a wavenumber consistent with the claim that energetically dominant magnetic structures have a size $\ell_{B} \sim 300 \mu \mathrm{m}$. The steeper slope of the spectrum at small wavelengths $(\$ 100 \mu \mathrm{m})$ is not a property of the true spectrum, but is due to diffusion of the imaging beam caused by small-scale magnetic fields, and the underestimation of the magnetic energy by the reconstruction algorithm in the presence of small-scale caustics (see Supplementary Methods for a discussion of these effects). In the FLASH simulations the magnetic field spectrum appears to be consistent with $\mathrm{a} \sim k^{-1}$ power-law dependence, as shown in Fig. 2d, in agreement with the spectra of tangled fields near and above the dynamo threshold found in ref. ${ }^{23}$ and by other investigators ${ }^{43-45}$, in the $\mathrm{Pm}<1$ regime.

Our experiment thus indicates that, as the two plasma flows collide, a strongly turbulent plasma, with magnetic Reynolds number above the threshold for dynamo action, is generated. The magnetic field grows from an initial value $B_{\text {rms }} \leqslant 4$ to $\sim 100-120$ $\mathrm{kG}$. We assume this to be near the saturated value because the Faraday rotation measurement begins over 2 ns (comparable to dynamical times) before the proton imaging diagnostic, and we infer similar magnetic field strengths from both. Note that the expected timescale for saturation to be reached is of the order of an outer-scale eddy-turnover time, $L / u_{L} \sim 6 \mathrm{~ns}$, a period that is comparable to the time that has elapsed between the initial flow collision and the magnetic field measurements. That the magnetized plasma is in a saturated state is corroborated by the FLASH simulation results (see Supplementary Figure 11 in Supplementary Methods).

If saturation is reached, the magnetic field energy should become comparable to the turbulent kinetic energy at the outer scale. We find $B_{\mathrm{rms}}^{2} / \mu_{0} \rho u_{L}^{2} \approx 0.04$ (where $\rho$ is the plasma mass density and we have taken $B_{\text {rms }} \approx 120 \mathrm{kG}$ ). Because the field distribution is expected to be quite intermittent and because $\mathrm{Rm}$ in our experiment is unlikely to be asymptotically large compared to the dynamo threshold value, it is reasonable that the mean magnetic energy density is quantitatively smaller than the kinetic energy density $10,11,17$. However, a good indication that the magnetic field has reached a dynamically saturated state is that it is dynamically strong in the most intense structures, which are not necessarily volume filling. To find an upper experimental bound on the maximum field, $B_{\max }$, we assume that the deflections acquired by the imaging protons across the plasma come from an interaction with a single structure. The strongest individual structure in the reconstructed path-integrated image has scale $\ell_{B} \sim 140 \mu \mathrm{m}$ with a path-integrated field of $6 \mathrm{kG} \mathrm{cm}$. This gives $B_{\max } \leqslant 430 \mathrm{kG}$, which leads to $B_{\max }^{2} /\left(\mu_{0} \rho u_{L}^{2}\right) \leqslant 0.5$, consistent with dynamical strength.

Our results appear to provide a consistent picture of magnetic field amplification by turbulent motions, in agreement with the longstanding theoretical expectation that turbulent dynamo is the dominant process in achieving dynamical equipartition between

Fig. 4 Proton radiography. a Normalized number of $15 \mathrm{MeV}$ protons detected on a CR-39 plate. The normalization is such that unity corresponds to the mean number of protons per pixel on the detector. The $D^{3} \mathrm{He}$ capsule was imploded at $t=29$ ns. Fusion reactions occur 0.6 ns after the start of the implosion and the protons are emitted isotropically within a short burst, of $\sim 150$ ps duration ${ }^{39}$. The flight time of the protons to the plasma is 0.1 ns. The chlorinated plastic foils were driven with a 10 ns long pulse shape (see Fig. 1). X-ray data and FLASH simulations indicate that the plasma flows are close to collision by 29 ns (see also Supplementary Figure 9 in Supplementary Methods). Thus, this proton image can provide an estimate of the initial seed fields. b Same as a, but with the deuterium-tritium capsule imploded at $t=34 \mathrm{~ns}$. The development of structures shows the development of fields in the interaction region. $\mathbf{c}$ Same as $\mathbf{b}$, but with the chlorinated plastic foils driven with the 5 ns long pulse, which gives higher flow velocities, and hence higher magnetic Reynolds numbers. $\mathbf{d}$ Reconstruction of magnetic fields for case $\mathbf{a}$. e Reconstruction of magnetic fields for case $\mathbf{b}$. $\mathbf{f}$ Reconstruction of magnetic fields for case $\mathbf{c}$. $\mathbf{g}$ Power spectrum of the magnetic energy from the reconstructed magnetic field from experimental data (the region bound by a dashed line in panel $\mathbf{f}$ ) 
kinetic and magnetic energies in high magnetic Reynolds number plasmas found in many astrophysical environments.

\section{Methods}

Experimental facility and diagnostics. The laser-driven experiments presented in this work were carried out at the Omega laser facility at the Laboratory for Laser Energetics of the University of Rochester ${ }^{32}$, under the auspices of the National Laser User Facilities (NLUF) program of the U.S. Department of Energy (DOE) National Nuclear Security Administration (NNSA). In order to fully characterize the plasma state and measure the magnetic field amplification we fielded a number of experimental diagnostics, including Thomson scattering, X-ray imaging, Faraday rotation, and proton radiography. Detailed discussions on each diagnostic, along with full descriptions on how the experimental measurements were analyzed, are given in Supplementary Methods of the Supplementary Information.

MHD and numerical simulations. The experimental platform was designed using radiation-MHD simulations with the publicly available, multi-physics code FLASH $^{46,47}$. The numerical modeling of the platform employed the entire suite of High Energy Density Physics capabilities ${ }^{33,34}$ of the FLASH code. We performed an extensive series of moderate-fidelity 2D cylindrical FLASH radiation-MHD simulations on the Beagle 2 cluster at the University of Chicago followed by a smaller set of high-fidelity 3D FLASH radiation-MHD simulations on the Mira supercomputer at the Argonne National Laboratory. The simulation campaign is described in detail in a companion paper ${ }^{34}$ and presented in Supplementary Methods. The applicability of the MHD approximation and considerations regarding the collisionality of the turbulent plasma are discussed in Supplementary Methods of the Supplementary Information.

Data availability. All data that support the findings of this study are available from the authors upon request.

Received: 23 June 2017 Accepted: 9 January 2018

Published online: 09 February 2018

\section{References}

1. Carilli, C. L. \& Taylor, G. B. Cluster magnetic fields. Annu. Rev. Astron. Astrophys. 40, 319-348 (2002).

2. Kouveliotou, C. et al. An x-ray pulsar with a superstrong magnetic field in the soft $\gamma$-ray repeater sgr1806- 20. Nature 393, 235-237 (1998).

3. Zweibel, E. G. \& Heiles, C. Magnetic fields in galaxies and beyond. Nature $\mathbf{3 8 5}$, 131-136 (1997).

4. Subramanian, K., Shukurov, A. \& Haugen, N. E. L. Evolving turbulence and magnetic fields in galaxy clusters. Mon. Not. R. Astron. Soc. 366, 1437-1454 (2006).

5. Schekochihin, A. A. \& Cowley, S. C. Turbulence, magnetic fields, and plasma physics in clusters of galaxies. Phys. Plasmas 13, 056501 (2006).

6. Ryu, D., Kang, H., Cho, J. \& Das, S. Turbulence and magnetic fields in the large-scale structure of the universe. Science 320, 909-912 (2008).

7. Miniati, F. \& Beresnyak, A. Self-similar energetics in large clusters of galaxies. Nature 523, 59-62 (2015).

8. Batchelor, G. K. On the spontaneous magnetic field in a conducting liquid in turbulent motion. Proc. R. Soc. Lond. Ser. A201, 405-416 (1950).

9. Biermann, L. \& Schlüter, A. Cosmic radiation and cosmic magnetic fields. II. Origin of cosmic magnetic fields. Phys. Rev. 82, 863-868 (1951).

10. Schekochihin, A. A., Cowley, S. C., Taylor, S. F., Maron, J. L. \& McWilliams, J. C. Simulations of the small-scale turbulent dynamo. Astrophys. J. 612, 276-307 (2004).

11. Beresnyak, A. Universal nonlinear small-scale dynamo. Phys. Rev. Lett. 108 035002 (2012).

12. Haugen, N. E., Brandenburg, A. \& Dobler, W. Simulations of nonhelical hydromagnetic turbulence. Phys. Rev. E 70, 016308 (2004).

13. Kulsrud, R. M., Cen, R., Ostriker, J. P. \& Ryu, D. The protogalactic origin for cosmic magnetic fields. Astrophys. J. 480, 481-491 (1997).

14. Stamper, J. A. et al. Spontaneous magentic fields in laser-produced plasmas. Phys. Rev. Lett. 26, 1012 (1971).

15. Gregori, G. et al. Generation of scaled protogalactic seed magnetic fields in laser-produced shock waves. Nature 481, 480-483 (2012).

16. Parker, E. E. N. Hydromagnetic dynamo models. Astrophys. J. 122, 293-314 (1955).

17. Meneguzzi, M., Frisch, U. \& Pouquet, A. Helical and nonhelical turbulent dynamos. Phys. Rev. Lett. 47, 1060-1064 (1981).

18. Kazantsev, A. Enhancement of a magnetic field by a conducting fluid. Sov. Phys. JETP 26, 1031-1034 (1968).
19. Krause, F. \& Raedler, K. H. Mean-Field Magnetohydrodynamics and Dynamo Theory (Pergamon Press, Oxford, 1980).

20. Zeldovich, I. B., Ruzmaikin, A. A. \& Sokoloff, D. D. in Magnetic Fields in Astrophysics Vol. 3 (Gordon and Breach Science Publishers, 1983), Translation.

21. Brandenburg, A., Sokoloff, D. \& Subramanian, K. Current status of turbulent dynamo theory. Space Sci. Rev. 169, 123-157 (2012).

22. Brandenburg, A. \& Lazarian, A. Astrophysical hydromagnetic turbulence. Space Sci. Rev. 178, 163-200 (2013).

23. Schekochihin, A. A. et al. Fluctuation dynamo and turbulent induction at low magnetic Prandtl numbers. N. J. Phys. 9, 300 (2007).

24. Kulsrud, R. M. Important plasma problems in astrophysics. Phys. Plasmas 2, 1735 (1995).

25. Gregori, G., Reville, B. \& Miniati, F. The generation and amplification of intergalactic magnetic fields in analogue laboratory experiments with high power lasers. Phys. Rep. 601, 1-34 (2015).

26. Monchaux, R. et al. Generation of a magnetic field by dynamo action in a turbulent flow of liquid sodium. Phys. Rev. Lett. 98, 044502 (2007).

27. Gailitis, A. et al. Detection of a flow induced magnetic field eigenmode in the riga dynamo facility. Phys. Rev. Lett. 84, 4365-4368 (2000).

28. Gailitis, A. et al. Magnetic field saturation in the riga dynamo experiment. Phys. Rev. Lett. 86, 3024-3027 (2001).

29. Ponomarenko, Y. B. Theory of the hydromagnetic generator. J. Appl. Mech. Tech. Phys. 14, 775-778 (1973).

30. Meinecke, J. et al. Turbulent amplification of magnetic fields in laboratory laser-produced shock waves. Nat. Phys. 10, 520-524 (2014).

31. Meinecke, J. et al. Developed turbulence and nonlinear amplification of magnetic fields in laboratory and astrophysical plasmas. Proc. Natl. Acad. Sci. 112, 8211-8215 (2015).

32. Boehly, T. et al. Initial performance results of the OMEGA laser system. Opt. Commun. 133, 495-506 (1997).

33. Tzeferacos, P. et al. FLASH MHD simulations of experiments that study shock-generated magnetic fields. High Energy Density Phys. 17, 24-31 (2015).

34. Tzeferacos, P. et al. Numerical modeling of laser-driven experiments aiming to demonstrate magnetic field amplification via turbulent dynamo. Phys. Plasmas 24, 041404 (2017)

35. Zhuravleva, I. et al. The relation between gas density and velocity power spectra in galaxy clusters: qualitative treatment and cosmological simulations. Astrophys. J. Lett. 788, L13 (2014).

36. Gaspari, M., Churazov, E., Nagai, D., Lau, E. T. \& Zhuravleva, I. The relation between gas density and velocity power spectra in galaxy clusters: highresolution hydrodynamic simulations and the role of conduction. Astron. Astrophys. 569, A67 (2014).

37. Evans, D. E. \& Katzenstein, J. Laser light scattering in laboratory plasmas. Rep. Prog. Phys. 32, 207-271 (1969).

38. Inogamov, N. A. \& Sunyaev, R. A. Turbulence in clusters of galaxies and x-ray line profiles. Astron. Lett. 29, 791-824 (2003).

39. Li, C. et al. Measuring E and B fields in laser-produced plasmas with monoenergetic proton radiography. Phys. Rev. Lett. 97, 3-6 (2006).

40. Kugland, N. L., Ryutov, D. D., Plechaty, C., Ross, J. S. \& Park, H. S. Invited Article: Relation between electric and magnetic field structures and their proton-beam images. Rev. Sci. Instrum. 83, 101301 (2012).

41. Graziani, C., Tzeferacos, P., Lamb, D. Q. \& Li, C. Inferring morphology and strength of magnetic fields from proton radiographs. Rev. Sci. Instrum. 88, 123507 (2017).

42. Bott, A. F. A. et al. Proton imaging of stochastic magnetic fields. J. Plasma Phys. 83 (2017)

43. Ruzmaikin, A. \& Shukurov, A. Spectrum of the galactic magnetic fields. Astrophys. Space Sci. 82, 397-407 (1982).

44. Kleeorin, N. \& Rogachevskii, I. Effective ampère force in developed magnetohydrodynamic turbulence. Phys. Rev. E 50, 2716-2730 (1994).

45. Kleeorin, N., Mond, M. \& Rogachevskii, I. Magnetohydrodynamic turbulence in the solar convective zone as a source of oscillations and sunspots formation Astron. Astrophys. 307, 293-309 (1996)

46. Fryxell, B. et al. FLASH: an adaptive mesh hydrodynamics code for modeling astrophysical thermonuclear flashes. Astrophys. J. Suppl. Ser. 131, 273-334 (2000).

47. Dubey, A. et al. Extensible component-based architecture for FLASH, a massively parallel, multiphysics simulation code. Parallel Comput. 35, 512-522 (2009)

\section{Acknowledgements}

The research leading to these results has received funding from the European Research Council under the European Community's Seventh Framework Programme (FP7/2007 2013)/ERC grant agreements no. 256973 and 247039, the U.S. Department of Energy under Contract No. B591485 to Lawrence Livermore National Laboratory, Field Work 
Proposal No. 57789 to Argonne National Laboratory, grants no. DE-NA0002724 and DE-SC0016566 to the University of Chicago, DE-NA0003539 to the Massachusetts Institute of Technology, and Cooperative Agreement DE-NA0001944 to the Laboratory for Laser Energetics University of Rochester. We acknowledge support from the National Science Foundation under grant PHY-1619573. This work was also supported in part by $\mathrm{NIH}$ through resources provided by the Computation Institute and the Biological Sciences Division of the University of Chicago and Argonne National Laboratory, under grant S10 RR029030-01. Awards of computer time were provided by the U.S. Department of Energy Innovative and Novel Computational Impact on Theory and Experiment (INCITE) and ASCR Leadership Computing Challenge (ALCC) programs. This research used resources of the Argonne Leadership Computing Facility at Argonne National Laboratory, which is supported by the Office of Science of the U.S. Department of Energy under contract DE-AC02-06CH11357. We acknowledge funding from grants 20070093860 and 2016R1A5A1013277 of the National Research Foundation of Korea. Support from AWE plc., the Engineering and Physical Sciences Research Council (grant numbers EP/M022331/1 and EP/N014472/1) and the Science and Technology Facilities Council of the United Kingdom is acknowledged.

\section{Author contributions}

This project was conceived by G.G., D.Q.L., P.T., B.R., and A.A.S. The experimental team was led by G.G. and the simulation work by P.T. The Thomson scattering polarimetry diagnostics at the Omega laser facility was designed by D.H.F. and J.K. C.-K.L. and R.P. contributed to the proton radiography development and data extraction. P.T., A.R., A.F. A.B., A.R.B., R.B., A.C., F.C., E.M.C., J.E., F.F., C.B.F., J.F., C.G., J.K., M.K., C.-K.L., J.M., R.P., H.-S.P., B.A.R., J.S.R., D.Ryu, D.Ryutov, T.G.W., B.R., F.M., A.A.S., D.Q.L., D.H.F., and G.G. have provided contributions to either data analysis, MHD simulations, or theoretical support.

\section{Additional information}

Supplementary Information accompanies this paper at https://doi.org/10.1038/s41467018-02953-2.

Competing interests: The authors declare no competing financial interests.

Reprints and permission information is available online at http://npg.nature.com/ reprintsandpermissions/

Publisher's note: Springer Nature remains neutral with regard to jurisdictional claims in published maps and institutional affiliations.

(c) Open Access This article is licensed under a Creative Commons Attribution 4.0 International License, which permits use, sharing, adaptation, distribution and reproduction in any medium or format, as long as you give appropriate credit to the original author(s) and the source, provide a link to the Creative Commons license, and indicate if changes were made. The images or other third party material in this article are included in the article's Creative Commons license, unless indicated otherwise in a credit line to the material. If material is not included in the article's Creative Commons license and your intended use is not permitted by statutory regulation or exceeds the permitted use, you will need to obtain permission directly from the copyright holder. To view a copy of this license, visit http://creativecommons.org/ licenses/by/4.0/.

(C) The Author(s) 2018 